\title{
Dose-dependent production of linoleic acid analogues in food derived Lactobacillus plantarum K25 and in silico characterization of relevant reactions
}

\author{
Tariq Aziz1, Abid Sarwar1, Muhammad Fahim², Jalal ud Din', Sam Al-Dalali', Zia Ud Din³, \\ Ayaz Ali Khan', Zhang Jian' ${ }^{4}$ and Zhennai Yang ${ }^{1 凶}$
}

${ }^{1}$ Beijing Advanced Innovation Center for Food Nutrition and Human Health, Beijing Technology and Business University, Beijing, China; ${ }^{2}$ Centre for Omic Sciences, Islamia College Peshawar, KP, Pakistan 25120; ${ }^{3}$ Department of Chemistry, Universidade Federeal de Sao Carlos, SP, Brazil; ${ }^{4}$ Department of Biotechnology, University of Malakand, KP, Pakistan, 18800

The objective of this study was to assess and scrutinize the competency of probiotic L. plantarum K25 to produce linoleic acid analogues in the medium supplemented with different concentrations of linoleic acid, ranging from $1 \%$ to $10 \%$, in a dose dependent manner. The analogues produced were identified and quantitated by GC-MS and in silico studies were done to confirm enzymatic reactions involved in its conversion. The results showed that $L$. plantarum K25 could convert linoleic acid at different concentrations to 9 different fatty acid analogues at concentrations ranging from 0.01 to 17.24 $\mathrm{mg} / \mathrm{L}$. Among these metabolites, formation of an essential fatty acid, the linolenic acid, in media supplemented with $9 \%$ linoleic acid, is being reported for the first time. Putative candidate enzymes involved in biotransformation of linoleic acid into linoleic acid analogues were identified in the whole genome of L. plantarum $\mathrm{K} 25$, which was sequenced previously. In silico studies confirmed that many enzymes, including linoleate isomerase and dehydrogenase, may be involved in biotransformation of linoleic acid into linoleic acid analogues. Both enzymes could effectively bind the linoleic acid molecule, mainly by forming hydrogen bonding between the acidic groups of linoleic acid and the proline residues at the active sites of the enzymes, validating putative reaction partners.

Key words: Lactobacillus plantarum, linoleic acid analogues, linoleate isomerase, dehydrogenase, in silico study

Received: 18 January, 2020; revised: 04 March, 2020; accepted: 04 March, 2020; available on-line: 18 March, 2020

⿶e-mail: yangzhennai@th.btbu.edu.cn

Acknowledgements of Financial Support: Financial support from the Natural Science Foundation of China (No. 31871823) and Beijing Talent Cultivation Quality Construction - First-class Professional Construction (Municipal Level) - Food Science and Engineering (PXM2019_014213_000010) is kindly acknowledged.

Abbreviations: CFŪs, colony forming units; CLA DH, dehydrogenase; CLA HY, hydratase; CLA, conjugated linoleic acid; CLA-DC, decarboxylase; CLA-ER, enoate reductase; El, electron ionization; FAME, fatty acid methyl esters; GC, gas chromatography; GC-MS, gas chromatography and mass spectrometry; GRAS, generally regarded as a safe; LA, linoleic acid; LAB, lactic acid bacteria; MS, mass spectra; NIST, National Institute of Standard and Technology Library; PUFA, polyunsaturated fatty acids

\section{INTRODUCTION}

Gut microorganisms are essential for maintaining their host's health by metabolizing dietary lipid into multiple fatty acid species, including conjugated fatty acids and trans-fatty acids. During the last 10 years, microbiologists have started exploring the role of gut microbes in lipid metabolism. Among various species, lactic acid bacteria (LAB) Lactobacillus plantarum has attained special attention to understand the lipid metabolism, especially in case of the saturation metabolism of polyunsaturated fatty acids. Lactobacillus plantarum is generally regarded as a safe (GRAS) microorganism and has been traditionally used in food fermentation. Many LAB strains are able to produce various bacteriocins (Nes et al., 2007), exopolysaccharides (Cerning et al., 1999; De Vuyst et al., 1999; Jiang et al., 2018) fatty acids, etc., exerting their beneficial health effects. Polyunsaturated fatty acids (PUFA), such as the linoleic acid (LA; 18:2 $\Delta 9 \mathrm{Z}, 12 \mathrm{Z}$ ), have inhibitory effects on growth of many bacteria (Greenway et al., 1979; Raychowdhury et al., 1985; Keweloh et al., 1996; Zheng et al., 2005). Increased concentrations of PUFA are toxic and may block native fatty acid biosynthesis via inhibition of the enoyl-ACP reductase (Greenway et al., 1979; Raychowdhury et al., 1985; Keweloh et al., 1996; Zheng et al., 2005). Bacteria have evolved a detoxifying mechanism and are able to enzymatically hydrogenate PUFA by a complete reduction of double bonds on the carbon chain, i.e. by biohydrogenation, producing nontoxic saturated fatty acids as the final product (Buccioni et al., 2010). This process involves various steps and has been best described for LA and oleic acid (OA; 18:1 $\triangle 9 Z$ ) that are converted to non-toxic saturated stearic acid (SA; 18:0) by the rumen microbiota (Buccioni et al., 2010; Maia et al., 2007; McKain et al., 2010; Nam et al., 2007). Lactobacillus plantarum is homofermentative with ability to transform the growth-inhibiting free polyunsaturated fatty acids, e.g. linoleic acid (LA), at a relatively high concentration, into bioactive conjugated LA (CLA) and other less toxic fatty acids metabolites, e.g. hydroxyfatty acids, oxo- fatty acids, conjugated fatty acids and saturated trans fatty acids, as intermediates to finally produce saturated monoenes (OA and trans-vaccenic acid) (Kishino et al., 2011a; Kishino et al., 2011b; Kishino et al., 2013).

Several enzymes that catalyze conversion of LA into a bioactive isomer of CLA have been biochemically characterized, e.g. linoleate isomerases derived from Butyrivibrio fibrisolvens (Kepler et al., 1971), Clostridium sporogenes (Peng et al., 2007), and Propionibacteruim acnes (Liavonchanka et al., 2006). Later, the Propionibacterium acnes isomerase was structurally characterized as a FAD dependent protein catalyzing production of 10-trans-12-cis-CLA (Liavonchanka et al., 2006; Liavonchanka et al., 2009). Furthermore, multiple proteins, such as a hydratase (CLA HY), 
dehydrogenase (CLA DH), decarboxylase (CLA-DC) and enoate reductase (CLA-ER) (S. Kishino et al., 2011a; S. Kishino et al., 2011b; S. Kishino et al., 2013), have been implicated in some biotransformation reactions. This saturation metabolism produces conjugated fatty acids and trans-fatty acids that are present in ruminant-derived foods with different physiological properties (Kishino et al., 2003).

Toxicity of the linoleic acid (LA; 18:2 $\Delta 9$ Z,12Z) and other PUFA leads to inhibition of bacterial growth and weakens cellular membranes by blocking native fatty acid biosynthesis through inhibition of the enoyl-ACP reductase (Greenway et al., 1979; Raychowdhury et al., 1985; Keweloh et al., 1996; Zheng et al., 2005). Certain bacteria have evolved a defense mechanism to override this toxicity by enzymatically hydrogenating PUFA, i.e. biohydrogenation. This is achieved by a complete reduction of double bonds on the carbon chain in PUFA, producing non-toxic saturated fatty acids as the final product (Buccioni et al., 2010). Our group previously reported that L. plantarum K25, a probiotic strain isolated from Tibetan kefir, has the ability to inhibit biofilm formation and decrease cholesterol levels (Zhang et al., 2013). Whole genome sequence analysis of $L$. plantarum K25 revealed that this strain has probiotic functionality as one of its plasmids, K25P2, has the ability to evade the immune system/ modulation of the immune system and nutritional stress and cold tolerance, and comprises of lysozyme, bile salt hydrolase, oxidoreductase, EPSs and terpenes (Yunyun Jiang et al., 2018). In silico analysis of the whole genome sequences of $L$. plantarum K25, revealed presence of several enzymes, including linoleate isomerase and dehydrogenase. Furthermore, the L. plantarum K25 strain harbors more genes that function in defense mechanisms and lipid transport metabolism, in comparison with other L. plantarum strains (Yunyun Jiang et al., 2018).

Here, we report that Lactobacillus plantarum K25 is resistant to LA toxicity at higher concentrations $(10 \%$ $\mathrm{w} / \mathrm{v}$ ) and that we were able to recover an essential fatty acid i.e., the linolenic acid from media supplemented with $9 \%$ LA, along with other LA metabolites at varying concentration in a (LA-) dose-dependent manner. We validated our findings by employing in silico analysis to characterize relevant reactions involving the enzymes that were identified by using the whole genome sequence of $L$. plantarum K25. Our findings have great prospect for human nutrition, nutraceuticals and food industry and will pave a way into identifying the relevant reactions and doses for biotransformation of LA into desirable LA-analogues.

\section{MATERIALS AND METHODS}

Microorganism and growth medium. L. plantarum K25 isolated and identified from Tibetan kefir grains by the Northeast Agricultural Research Center of China, Changchun, China was maintained in the culture bank of the Dairy Laboratory at the Beijing Technology and Business University. L. plantarum K25 was revived consecutively 3 times at $37^{\circ} \mathrm{C}$ in the MRS medium containing $1.0 \%$ tryptone, $1.0 \%$ meat extract, $0.5 \%$ yeast extract, $2.0 \%$ glucose, $0.1 \%$ Tween $80,0.2 \% \mathrm{~K}_{2} \mathrm{HPO}_{4}$, $0.5 \%$ sodium acetate, $0.2 \%$ diammonium citrate, $0.02 \%$, $\mathrm{MgSO}_{4} \times 7 \mathrm{H}_{2} \mathrm{O}$ and $0.005 \%, \mathrm{MnSO}_{4} \times \mathrm{H}_{2} \mathrm{O}$. All medium components were dissolved in distilled water, adjusted to $\mathrm{pH} 5.5$ and sterilized at $121^{\circ} \mathrm{C}$ for 15 minutes. Fresh MRS medium with added different concentrations of LA was inoculated with $1 \%$ of the activated culture of $L$. plantarum K25 for growth and production of LA analogues at $37^{\circ} \mathrm{C}$.

Growth of L. plantarum K25 for production of LA analogues. The MRS medium was supplemented with linoleic acid (during growth of $L$. plantarum K25 in the MRS medium containing $1 \%, 2 \%, 3 \%, 4 \%, 5 \%$, $6 \%, 7 \%, 8 \%, 9 \%$ and $10 \%$ (w/v) of LA obtained from the Shanghai yuanye Bio-Technology Co. Ltd with 99\% purity), culture samples were taken at $0,8,16,24,36$ and $48 \mathrm{~h}$ for determination of viable counts by the plate count method, as expressed by colony forming units (CFUs) per $\mathrm{mL}$, and for determination of LA analogues as described below.

Spectrophotometric determination of LA analogues. The culture sample were centrifuged $(13000 \times g$ for $5 \mathrm{~min}$ at $4^{\circ} \mathrm{C}$ ), and $1 \mathrm{~mL}$ of the supernatant was mixed with $2 \mathrm{~mL}$ of isopropanol. After addition of 1.5 $\mathrm{mL}$ of hexane, the mixture was thoroughly vortexed in order to extract the lipids and then allowed to stand for $5 \mathrm{~min}$. The hexane layer was collected and the absorbance was measured at $233 \mathrm{~nm}$. The LA analogues were extracted by using hexane/isopropanol (2:1, v/v) solution at room temperature, and the extracts were washed with distilled water and then dehydrated with anhydrous sodium sulfate (Barrett et al., 2007).

Extraction of fatty acids from the medium. For analysis by gas chromatography (GC), the culture samples were centrifuged at $1900 \mathrm{rpm}$ for 5 minutes at $4^{\circ} \mathrm{C}$ to remove the cells. An internal standard (C17:0, heptadecanoic acid, 98\% pure; Macklin) was added to $5 \mathrm{ml}$ of the supernatant fluid to give a final concentration of $0.15 \mathrm{~g} / \mathrm{ml}$. Then, $5 \mathrm{~mL}$ of isopropanol were added and vortexed for $30 \mathrm{~s}$. Subsequently $2 \mathrm{~mL}$ of isopropanol were added and vortexed for $30 \mathrm{~s}$. Finally, $5 \mathrm{~mL}$ of nhexane were added to this mixture, vortexed for $3 \mathrm{~min}$, incubated for $30 \mathrm{~min}$, and centrifuged at $1900 \mathrm{rpm}$ for 5 min. The upper hexane layer containing fatty acid methyl esters (FAME) was collected and dried under a steam of liquid nitrogen (Shantha et al., 1993).

Gas chromatography and mass spectrometry (GCMS). The GC-MS analysis was done by using Shimadzu GC-2010 instrument coupled with a Dual Stage TMP (Ultra) mass spectrometer. The FAME sample of $2 \mu \mathrm{L}$ was injected in a split mode, set at 10:1 split ratio at $250^{\circ} \mathrm{C}$. The carrier gas was helium at a constant flow rate of $1 \mathrm{~mL} / \mathrm{min}$. The separation was conducted on a highly polar (TR-Wax MS, $30 \mathrm{~m}$ length $\times 0.25 \mathrm{~mm}$ i.d. $\times 0.25 \mu \mathrm{m}$ thickness) and fused silica capillary column (Thermo Fisher Scientific). The initial oven temperature was held at $170^{\circ} \mathrm{C}$ for $1 \mathrm{~min}$, then increased at $0.8^{\circ} \mathrm{C} /$ $\min$ to $200^{\circ} \mathrm{C}$. The temperature of line transfer was at $250^{\circ} \mathrm{C}$, and the ion source was controlled at $200^{\circ} \mathrm{C}$. The MS detector was operated in an electron ionization (EI) voltage of $70 \mathrm{eV}$ under a mass scan range of 33-450 amu $(m / z)$.

Identification and quantitation of LA analogues

Chemical identification was conducted by comparison of the mass spectra (MS) of the peaks with those found in the National Institute of Standard and Technology library (NIST, 2014).

Quantitation analysis was conducted by comparison of the respective peak area with that of the internal standard (i.e. heptadecanoic acid $50 \mathrm{mg} / 5 \mathrm{~mL}$ of isopropanol). The concentration of each compound was calculated depending on the follwing equation:

Concentration of volatiles $=\frac{\text { peak area of the compound }}{\text { peak area of the internal standard }} \times$ concentration of the internal standard 
All of the samples were analyzed in triplicate.

Genome assembly and annotation. Previously, our research group has extracted genomic DNA using Wizard ${ }^{\circledR}$ Genomic DNA Purification Kit (Promega) according to the manufacturer's protocol. Purified genomic DNA was quantified by TBS-380 fluorometer (Turner Bio Systems Inc., Sunnyvale, CA). High quality DNA (OD260/280=1.8 2.0, >20 $\mu \mathrm{g}$ ) was used and libraries were made with the SMRT Bell gDNA protocol. Genomic DNA sequenced with a PacBio RS II platform, yielding 44144 reads (Jiang et al., 2018), was further used in this research. The low quality reads were filtered and afterwards the filtered reads were assembled by SMRT Link (http://www.pacb.com/wpcontent/uploads/SMRT_Link_User_Guide.pdf), which consisted of Sample Setup, Run Design, Run QC, Data Management and SMRT Analysis modules to generate 7 contigs representing one chromosome and 6 plasmids without gaps (Overbeek et al., 2014; Koren et al., 2015; Berlin et al., 2015). The RAST online server (http:// www.rast.nmpdr.org/) (Koren et al., 2015) was used for the prediction of protein coding sequences (CDSs), functional annotation of predicted genes and identification of tRNA and rRNA..

In silico characterization of conversion of LA into LA analogues by L. plantarum K25. Enzymes catalyzing the relevant reactions for conversion of LA to its fatty acid analogues were identified by analysis of the whole genome sequence (https://www.ncbi.nlm.nih. gov/assembly/GCA_003020005.1) of L. plantarum K25, previously reported by our group by using the SWISSMODEL that is a fully automated protein structure homology modelling server (Biasini et al., 2014). The protein module was visualized by protein visualizing program Discovery Studio 3.5 (Dassault Systèmes BIOVIA, Discovery Studio Modeling Environment, 2015). The LA molecule was drawn in ChemSketch v15.0.9 (Chemaxon) assigned with proper $2 \mathrm{D}$ orientation, and the structure of each compound was analyzed for connection error in bond order. Energy of the molecules was minimized using Avogadro (Hanwell et al., 2012) with MMFF94 force field. Docking studies calculations were performed with the Autodock Tool (Morris et al., 2009). Protein (oxidoreductase) and ligands (LA) structures were converted to pdbqt file by MGL Tools 1.5.6 $\mathrm{rc}^{3}$. The interactions of complex protein-ligand conformations were analyzed using Autodock Tools 4.2 (Morris et al., 2009) and Discovery Studio 4.1 (Dassault Systèmes BIOVIA, Discovery Studio Modeling Environment, 2015).

Statistical Analysis. Data were statistically analyzed by ANOVA SAS version 9. Evaluation of the significance of differences between groups was performed with one-way ANOVA, as mentioned in the figure legends.

\section{RESULT AND DISCUSSION}

\section{Tolerance to linoleic acid concentration by $L$. plantarum K25 and production of LA analogues}

In the study presented here, the LA tolerance was evaluated by addition of increasing fatty acid concentrations in a range of $1 \%$ to $10 \%$, to MRS agar plates spread with L. plantarum K25. In our studies, L. plantarum K25 exhibited identical growth pattern at the concentrations of LA ranging from $1 \%(\mathrm{w} / \mathrm{v})$ to $10 \%$ $(\mathrm{w} / \mathrm{v})$, as shown in Fig $1 \mathrm{a}$. For all the concentrations of LA tested, it was observed that L. plantarum K25 had increased its growth up to $24 \mathrm{~h}$, subsequently entered

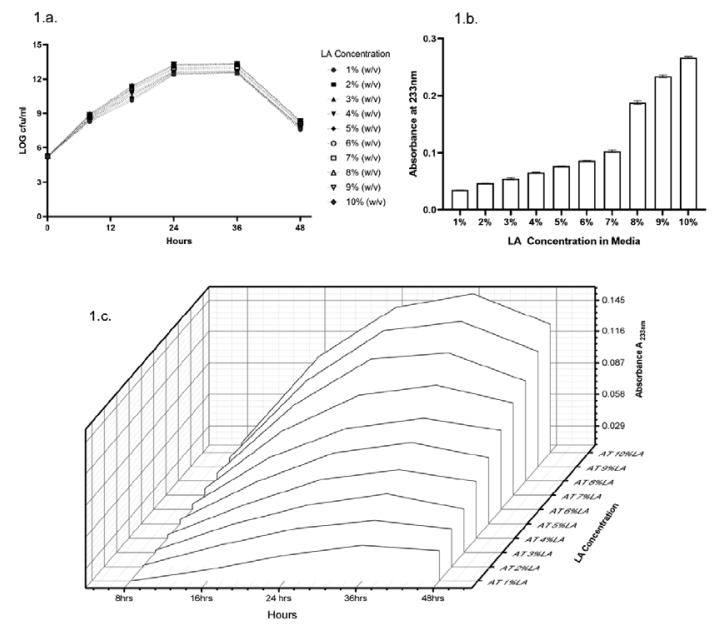

Figure 1. Tolerance of Lactobacillus plantarum K25 and production of LA analogues:

(a). Growth pattern of L. plantarum K25 as affected by different concentrations of linoleic acid, using the plate count method at $0,8,16,24,36$ and $48 \mathrm{~h}$ respectively. (b) Linoleic acid analogues produced by L. plantarum K25 with different concentrations of LA in the medium as determined by a spectrophotometric method measuring absorbance at $233 \mathrm{~nm}$. (c) Linoleic acid analogues produced at $8,16,24,36$ and $48 \mathrm{~h}$ during growth of L. plantarum K25 at different concentrations of LA in the medium.

the stationary phase till $36 \mathrm{~h}$, and then the growth decreased till $48 \mathrm{~h}$. There was a slight increase in the growth of the strain with the increase of the LA concentration. On the contrary, many other LAB strains were reported to be inhibited to different extent by LA and their tolerance to LA varied (Jiang et al., 1998; Barrett et al., 2007; Chaia et al., 2007; Ogawa et al., 2005). Jiang et al (1998) and many other researchers have reported that LA has an inhibitory effect on bacterial growth with varying level of tolerance according to strain. While earlier studies showed that even lower LA levels $(25 \mu \mathrm{g} / \mathrm{ml} \approx 1 \%)$ could inhibit bacterial growth, $L$. plantarum K 25 was able to grow well at the concentration of LA up to $10 \%(\mathrm{w} / \mathrm{v})$, indicating its relatively high tolerance to LA. Similarly, Chaia et al (2007) reported that certain bacteria i.e., Lactobacillus casei, Lactobacillus rhamnosus, Bifidobacterium bifidum and Streptococcus thermophiles were able to grow at a high concentration $(100 \mu \mathrm{g} / \mathrm{ml})$ of LA. However, the mechanism of tolerance is unclear for most of the bacteria reported.

Production of all LA analogues had gradually increased with the increase of LA concentration from $1 \%$ $(\mathrm{w} / \mathrm{v})$ to $10 \%(\mathrm{w} / \mathrm{v})$, as indicated by the increased absorption at the wavelength of $233 \mathrm{~nm}$ (Fig. 1b). This indicated that L. plantarum K25 exhibited high tolerance to LA by converting LA to less toxic LA analogues. Previous studies also advocated that conversion of free LA to LA analogues might function as a detoxification mechanism in bacteria and a stronger LA tolerance indicated a higher productivity in LA analogues (Shantha et al., 1993; Chaia et al., 2007; Chung et al., 2008; Lee et al., 1999). Furthermore, more LA analogues were produced during the stationary phase of growth of $L$. plantarum K25 and this production was reduced when the death phase started. Similar findings were also reported for other microbial producers of LA analogues (L. plantarum AKU 1009a) (Kishino et al., 2011; Alonso et al., 2003). Different LA analogues produced by $L$. plantarum K25 at different concentrations of LA in the medium, at time interval of $8,16,24,36$ and 48 hours are shown in Fig 1c. 
Table 1. Production (mg/L) and identification of LA analogues by L. plantarum $\mathrm{K} 25$ in the medium supplemented with different concentrations of LA.

\begin{tabular}{|c|c|c|c|c|c|c|c|c|c|c|c|}
\hline \multirow[b]{2}{*}{ LA analogues } & \multirow[b]{2}{*}{$\begin{array}{l}\text { Retention } \\
\text { Time (min) }\end{array}$} & \multicolumn{10}{|c|}{ Concentration of LA $(w / v)$} \\
\hline & & $1 \%$ & $2 \%$ & $3 \%$ & $4 \%$ & $5 \%$ & $6 \%$ & $7 \%$ & $8 \%$ & $9 \%$ & $10 \%$ \\
\hline 1. Ethyl Oleate & 15.28 & $\begin{array}{l}0.09^{f} \\
\pm 0.007\end{array}$ & $\begin{array}{l}0.16 \mathrm{e} \\
\pm 0.01\end{array}$ & $\begin{array}{l}0.25^{b} \\
\pm 0.01\end{array}$ & $\begin{array}{l}0.19 \mathrm{~d} \\
\pm 0.007\end{array}$ & $\begin{array}{l}0.26^{\mathrm{b}} \\
\pm 0.007\end{array}$ & $\begin{array}{l}0.22^{c} \\
\pm 0.02\end{array}$ & $\begin{array}{l}0.28^{\mathrm{b}} \\
\pm 0.004\end{array}$ & $\begin{array}{l}0.26^{\mathrm{b}} \\
\pm 0.001\end{array}$ & $\begin{array}{l}0.19 \mathrm{~d} \\
\pm 0.01\end{array}$ & $\begin{array}{l}0.34^{\mathrm{a}} \\
\pm 0.1\end{array}$ \\
\hline $\begin{array}{l}\text { 2. (E)-9-Octadecenoic acid } \\
\text { ethyl ester }\end{array}$ & 15.38 & n.d $d^{b}$ & n.d $d^{b}$ & $n \cdot d^{b}$ & $n \cdot d^{b}$ & n.d $d^{b}$ & n.d $d^{b}$ & n.d $d^{b}$ & n.d $d^{b}$ & $\begin{array}{l}0.01^{\mathrm{a}} \\
\pm 0.0001\end{array}$ & $\begin{array}{l}0.01^{\mathrm{a}} \\
\pm 0.0004\end{array}$ \\
\hline $\begin{array}{l}\text { 3. trans,trans-9,12-Octadeca- } \\
\text { dienoic acid, propyl ester }\end{array}$ & 15.68 & $\mathrm{n} \cdot \mathrm{d}^{\mathrm{b}}$ & n.d $d^{b}$ & $n \cdot d^{b}$ & n.d $d^{b}$ & n.d $d^{b}$ & n.d $d^{b}$ & n.d $d^{b}$ & n.d $d^{b}$ & $\begin{array}{l}0.01^{\mathrm{a}} \\
\pm 0.0007\end{array}$ & $\begin{array}{l}0.02^{\mathrm{a}} \\
\pm 0.003\end{array}$ \\
\hline 4. Octadecanoic acid & 25.68 & n.de & n.de & n.de & n.de & n.de & n.de & $\begin{array}{l}2.02^{\mathrm{a}} \\
\pm 0.15\end{array}$ & $\begin{array}{l}0.09 \mathrm{~b} \\
\pm 0.007\end{array}$ & $\begin{array}{l}0.05^{c} \\
\pm 0.008\end{array}$ & $\begin{array}{l}0.03^{\mathrm{d}} \\
\pm 0.001\end{array}$ \\
\hline 5. 6-Octadecenoic acid & 30.28 & n. $d^{h}$ & $\begin{array}{l}1.62^{\mathrm{g}} \\
\pm 0.1\end{array}$ & $\begin{array}{l}2.41^{d} \\
\pm 0.11\end{array}$ & $\begin{array}{l}1.88^{f} \\
\pm 0.12\end{array}$ & $\begin{array}{l}2.72^{c} \\
\pm 0.23\end{array}$ & $\begin{array}{l}2.14 \mathrm{e} \\
\pm 0.81\end{array}$ & $\begin{array}{l}2.81^{c} \\
\pm 0.25\end{array}$ & $\begin{array}{l}3.15^{b} \\
\pm 0.65\end{array}$ & $\begin{array}{l}2.20 \mathrm{e} \\
\pm 0.92\end{array}$ & $\begin{array}{l}3.85^{\mathrm{a}} \\
\pm 0.49\end{array}$ \\
\hline 6. 9-Octadecenoic acid & 30.25 & $\begin{array}{l}0.68^{\mathrm{a}} \\
0.007\end{array}$ & n.d $d^{b}$ & $n \cdot d^{b}$ & $n \cdot d^{b}$ & $n \cdot d^{b}$ & $n \cdot d^{b}$ & n.d $d^{b}$ & n.d $d^{b}$ & n.d $d^{b}$ & n.d $d^{b}$ \\
\hline $\begin{array}{l}\text { 7. 9,12-Octadecadienoic acid } \\
(\mathbf{Z}, \mathbf{Z})-\end{array}$ & 32.96 & n.d $d^{b}$ & $\begin{array}{l}17.24^{\mathrm{a}} \\
\pm 1.5\end{array}$ & $n \cdot d^{b}$ & $n \cdot d^{b}$ & n.d $d^{b}$ & n.d $d^{b}$ & n.d $d^{b}$ & n.d $d^{b}$ & n.d $d^{b}$ & n.d $d^{b}$ \\
\hline 8. Linoelaidic acid & 32.99 & n.de & n.de & n.de & n.de & n.de & $\begin{array}{l}9.03 c \\
\pm 1.12\end{array}$ & $\begin{array}{l}9.73 a \\
\pm 0.45\end{array}$ & $\begin{array}{l}9.37 \mathrm{~b} \\
\pm 0.78\end{array}$ & $\begin{array}{l}8.08^{d} \\
\pm 0.85\end{array}$ & $\begin{array}{l}8.09 \mathrm{~d} \\
\pm 1.3\end{array}$ \\
\hline 9.Linolenic acid & 36.22 & $n \cdot d^{b}$ & $n \cdot d^{b}$ & $n \cdot d^{b}$ & $n \cdot d^{b}$ & n.d $d^{b}$ & n.d $d^{b}$ & $n \cdot d^{b}$ & n.d $d^{b}$ & $\begin{array}{l}0.02^{\mathrm{a}} \\
\pm 0.001\end{array}$ & $\begin{array}{l}0.03^{\mathrm{a}} \\
\pm 0.001\end{array}$ \\
\hline
\end{tabular}

${ }^{*}$ n.d: not detected. Different letters superscripted shows statistical variences within a row.

\section{Identification and quantitation of LA analogues by GC-MS}

The LA analogues produced by L. plantarum K25 at different concentrations of LA were identified and quantitated by GC-MS (Table 1 and Fig 2). In total, 9 LA analogues were identified, including one saturated fatty acid, octadecanoic acid $\mathrm{C}_{18} \mathrm{H}_{36} \mathrm{O}_{2}$, two mono-unsaturated fatty acids, 6-octadecenoic acid $\mathrm{C}_{18} \mathrm{H}_{34} \mathrm{O}_{2}$ and 9-octadecenoic acid $\mathrm{C}_{18} \mathrm{H}_{34} \mathrm{O}_{2}$, two isomers of $\mathrm{LA}$, linoelaidic acid $\mathrm{C}_{18} \mathrm{H}_{32} \mathrm{O}_{2}$ and 9,12-octadecadienoic acid (Z,Z) $\mathrm{C}_{18} \mathrm{H}_{32} \mathrm{O}_{2}$; three fatty acid esters, ethyl oleate $\mathrm{C}_{20} \mathrm{H}_{38} \mathrm{O}_{2}$, (E)-9-octadecenoic acid ethyl ester $\mathrm{C}_{20} \mathrm{H}_{38} \mathrm{O}_{2}$ and trans, trans-9,12octadecadienoic acid $\mathrm{C}_{18} \mathrm{H}_{32} \mathrm{O}_{2}$, propyl ester; and one
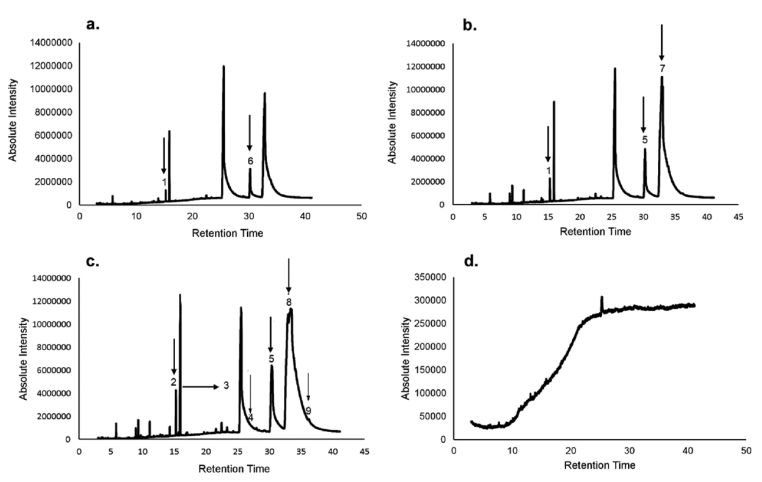

Figure 2. Gas chromatography of representative culture samples showing peaks of the LA analogues produced by L. plantarum K25.

(a) $1 \%$ of added LA; (b) $5 \%$ of added LA (c) $10 \%$ of added LA and Control (without LA). The metabolites/analogues produced by L. plantarum K25 under different concentrations of LA supplemented into MRS media include ethyl oleate, (E)-9-octadecenoic acid ethyl ester, trans, trans-9,12-octadecadienoic acid, propyl ester, octadecanoic acid, 6-octadecenoic acid, 9-octadecenoic acid, 9,12-octadecadienoic acid (Z, Z)-, linoelaidic acid and linolenic acid. omega-3 fatty acid $\mathrm{C}_{60} \mathrm{H}_{92} \mathrm{O}_{6}$, linolenic acid $\mathrm{C}_{18} \mathrm{H}_{32} \mathrm{O}_{2}$. The GC chromatograms of the representative samples showed 9 peaks corresponding to the 9 LA analogues at different retention time, ranging from $15.28 \mathrm{~min}$ for ethyl oleate to 36.22 min for linolenic acid.

Quantitation analysis of these peaks showed that L. plantarum K25 produced different amounts of LA analogues, ranging from $0.01 \mathrm{mg} / \mathrm{L}$ for (E)-9-octadecenoic acid ethyl ester or trans, trans-9,12-octadecadienoic acid, propyl ester, to $17.24 \mathrm{mg} / \mathrm{L}$ for 9,12-octadecadienoic acid $(\mathrm{Z}, \mathrm{Z})$ (Table 1). The $9 \mathrm{LA}$ analogues were also found to be produced at varying concentrations of LA, though the produced amounts were not dependent on the concentration of LA. Ethyl oleate could be produced by L. plantarum K25 from 1\% of LA at $0.09 \mathrm{mg} / \mathrm{L}$ to $10 \%$ of LA at $0.34 \mathrm{mg} / \mathrm{L}$. Another LA analogue, 6-octadecenoic acid, was also produced at a wide range of LA concentrations ranging from $2 \%$ at $1.62 \mathrm{mg} / \mathrm{L}$ to $10 \%$ at $3.85 \mathrm{mg} / \mathrm{L}$. However, 9-octadecenoic acid was produced only at $1 \%$ of LA at $0.68 \mathrm{mg} / \mathrm{L}$, while 9,12 -octadecadienoic acid $(\mathrm{Z}, \mathrm{Z})$ was produced at $2 \%$ of LA at the highest amount of $17.24 \mathrm{mg} / \mathrm{L}$. Two esters of (E)-9-octadecenoic acid ethyl ester and trans, trans-9,12octadecadienoic acid propyl ester, octadecanoic acid and linoelaidic acid were produced from $7 \%$ to $10 \%$ and $6 \%$ to $10 \%$ of $\mathrm{LA}$, at 0.03 to $2.02 \mathrm{mg} / \mathrm{L}$ and 8.08 to 9.73 $\mathrm{mg} / \mathrm{L}$, respectively.

Interestingly, we found that an essential fatty acid, the linolenic acid, was produced in media supplemented with higher concentrations $(9 \%$ and $10 \%)$ of LA, at a concentration of 0.01 to $0.02 \mathrm{mg} / \mathrm{L}$. Previously, L. plantarum strains isolated from the gut were reported to contain genes encoding enzymes responsible for saturation of unsaturated fatty acids to produce many intermediates, including the oxo-fatty acids, hydroxyl fatty acids, and partially saturated fatty acids (Kishino et al., 2013). It is also reported that LA was produced by Clostridium bifermentans JCM 1386 by saturation of arachidonic acid, a 20-carbon polyunsaturated fatty acids 


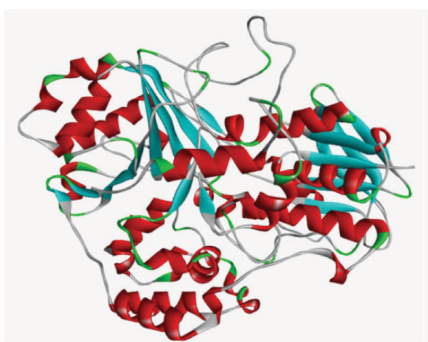

(a)

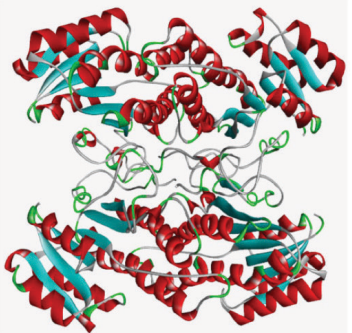

(b)
Figure 3. Tertiary structure of two putative enzymes involved in conversion of LA to LA analogues:

(a) linoleate isomerase, (b) dehydrogenase.

(PUFA) during anaerobic cultivation. However, L. plantarum strain K25 is also capable of producing linolenic acid, an essential fatty acid, from an 18-carbon PUFA (LA) as a substrate, probably due to the action of a dehydrogenase, as shown in the following in silico studies (Sakurama et al., 2014). Kepler and Tove (1967) reported that Butyrivibrio fibrisolvens could isomerize LA and linolenic acid to their corresponding conjugated acids, and further hydrogenation of the formed conjugated trienoic acid could produce a di-unsaturated fatty acid. Butyrivibrio fibrisolvens could also hydrogenate different geometrical isomers of linoleic acid to form conjugated LA, but it did not hydrogenate the trans-9-octadecenoic acid, trans-11-octadecenoic and cis-9-octadecenoic acid isomers (Kishino et al., 2011). However, the LA analogues that were produced by $L$. plantarum K25 from LA involving dehydrogenation, isomerization and reduction were further studied by in silico calculation as described in the following section.

\section{In silico calculation for relevent reactions (dehydrogenation, isomerisation, reduction and desertification)}

For conversion of LA to its fatty acid analogues by L. plantarum $\mathrm{K} 25$, the most prominent reactions included dehydrogenation, isomerization and reduction. Two main putative enzymes were identified as linoleate isomerase and dehydrogenase (Fig. 3) using the whole genome sequence (https://www.ncbi.nlm.nih.gov/assembly/ GCA_003020005.1) of L. plantarum K25 (Yunyun Jiang et al., 2018). LA was converted to its respective analogues by the enzymatic machinery of L. plantarum K25.

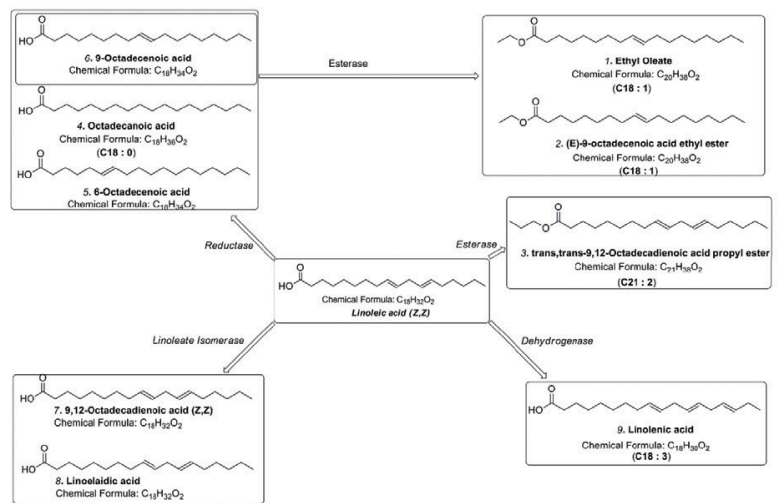

Figure 4. Conversion of LA into the different LA analogues by $L$. plantarum K25 involving enzymatic reactions, including dehydrogenation, isomerization, reduction and desertification.

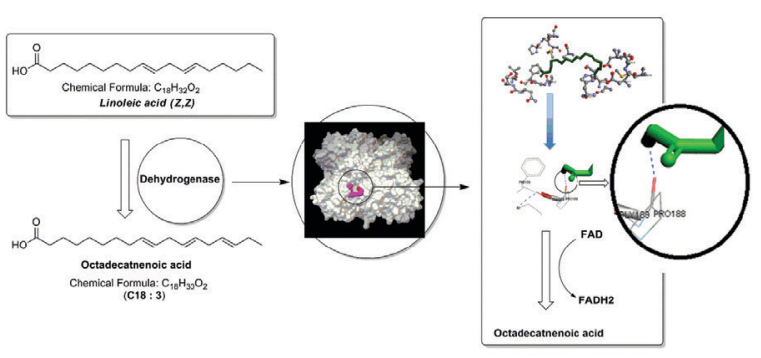

Figure 5. showing in silico depiction of dehydrogenation of LA to form lenolenic acid via enzyme dehydrogenase and active site of the molecule.

The main enzymatic reactions observed are isomerization, hydrogenation, dehydrogenation and formation of an ester group.

The enzymes responsible for different reactions by $L$. plantarum K25 strain, in which the conversion of LA to the different LA analogues occurred due to the enzymatic reactions including dehydrogenation, isomerization, reduction and desertification are shown in Fig. 4.

\section{Dehydrogenation}

To our knowledge, this is the first report of biotransformation of LA into linolenic acid by L. plantarum K25. The in silico analysis revealed that the FAD-dependent enzyme, dehydrogenase, plays a vital role in this dehydrogenation process. As visualized, the LA complex was docked with the dehydrogenase, and the active site of the enzyme perfectly held the LA molecule by electrostatic interaction and formation of hydrogen bonding between the acidic group of LA and the proline-188 residue of the enzyme in a lock and key model (Fig. 5).

In silico depiction of dehydrogenation of LA to form linolenic acid that involves the enzyme dehydrogenase with its active site holding the molecule of LA in a lock and key model by formation of hydrogen bonding between the acidic group of LA and the proline residue of the enzyme. The hydrogen bonding interaction between the acidic group of LA and the proline 188 residue is enlarged in the figure. Hydrogen bonding is important for the dehydrogenation reaction as it facilitates the reaction of FAD with substrate. After mechanistic reaction of enzyme, the FAD is converted to FADH2 leading to formation of a new double bond in the substrate (linoleic acid). Dehydrogenation in this case is FAD dependent. because after reaction FAD is converted into FADH2. The amino acid residues involved in this dehydrogenation reaction are those in the active site, including but not limited to Ala94, Phe190, Gly189, Pro188, Gly92, Ala91, His16, Gly13, Asp37, Met59, Ile38 and Asp63.

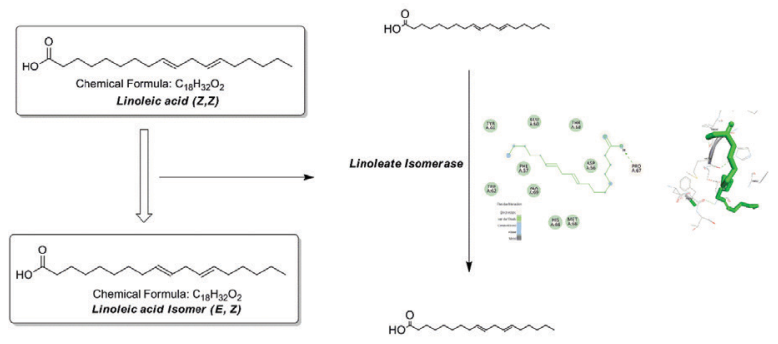

Figure 6. Conversion of the $Z, Z$ isomer of $L A$ to the $E, Z$ isomer by $L$. plantarom K 25 via enzyme Linoleate Isomerase. 

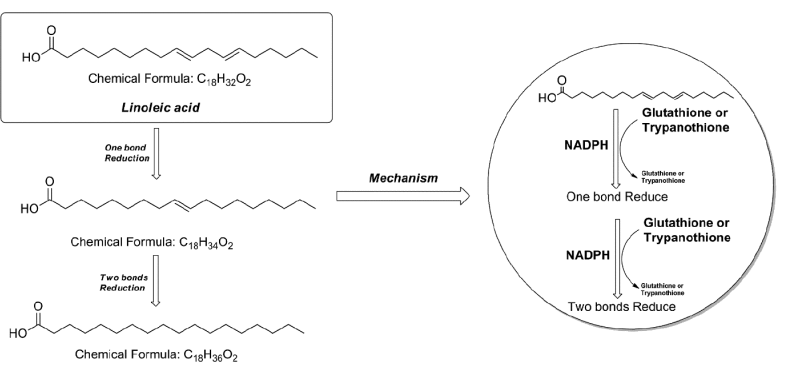

Figure 7. Reduction of LA by L. plantarum K25 to form 6-octadecenoic acid and 9-octadecenoic acid with one double bond reduced, and 9-octadecenoic acid with both double bonds reduced.

\section{Isomerisation}

In silico analysis revealed that conversion of the $\mathrm{Z}, \mathrm{Z}$ isomer of LA into the E,Z isomer by L. plantarum K25 is catalyzed by the linoleate isomerase. The LA molecule fits into the active site of the isomerase, and the acidic group of LA forms hydrogen bonding with the amino acid residue proline 67. The van der waal interaction is present between LA and the amino acid residues Tyr-61, Glu-60, Thr-58, Asp-56, Trp-62, Phe-57, Ala-69, His-66 and Met-68. The linoleate isomerase is also a NADPH dependenet enzyme which reduces the double bond of LA into a single bond, and reversiblly the double bond is formed with the inversion of configuration (Fig. 6). The acidic part is held by the enzyme through H-bonding, which faciliatates the action of formation of a double bond. The biocatalytic formation of double bond through trans-manner is the selectivness of using micro organism for the purpose.

Conversion of the Z,Z isomer of LA to the E,Z isomer by $L$. plantarom K25 as catalyzed by the linoleate isomerase with its actve site that binds the LA molecule by forming hydrogen bonding between the acidic group of LA and the amino acid residue proline 67 , and the van der waal interaction between LA and the amino acid residues Tyr-61, Glu-60, Thr-58, Asp-56, Trp-62, Phe57, Ala-69, His-66 and Met-68. The hydrogen bonding between the acidic group of LA and Pro 67 are encircled in red in the figure.

\section{Reduction}

All living cells, including L. plantarum K25, contain potential nucleophiles, like glutathione and trypanothione, which can reduce the double bond of LA to produce two types of compounds having one double bond reduced, such as the 6-octadecenoic acid and 9-octadecenoic acid, and both double bonds reduced, such as the 9-octadecanoic acid, employing a well know reduction mechanism involving NADPH, as shown in Fig 7. In the first step, the potential nucleophile glutathione and trypanothione formed a complex with LA, and in the second step reduction occurs by the action of NADPH. The LA and nucleophile complex is very unstable and remains for a fraction of time and simultaneously the reduction takes place. The NADPH is converted back to NADP, leading to conversion of LA into its reduced form. The potential of Lactobacillus is to control the reduction reaction, which is difficult to control by applying a synthetic reagent to only reduce one bond. That is why enzymatic/biotransformation is a strong method to use it for selective reduction.

\section{Formation of esters}

It is known that living cells serve as a factory for formation of many chemicals, including amino acids, potential nucleophiles and alcohols. In L. plantarum K25, formation of ethanol and propanol in the cells provided the mechanistic pathway for the esterification reaction. The enzymatic assembly of the bacterial cells helped the reaction of LA with ethanol and propanol to produce ethyl and propyl esters. Moreover, isopropanol, which was present in the growth medium, was also used in the reaction to form esters by $L$. plantarum K25.

\section{CONCLUSION}

Probiotic L. plantarum K25 was shown to be capable of converting LA, ranging from $1 \%$ to $10 \%$ (w/v), to various LA analogues, such as the octadecanoic acid; 6-octadecenoic acid; 9-octadecenoic acid; linoelaidic acid; 9,12-octadecadienoic acid (Z, Z); ethyl oleate; (E)-9-octadecenoic acid ethyl ester; trans, trans-9,12-octadecadienoic acid, propyl ester; and linolenic acid. The main reactions involved in this conversion include isomerization, dehydrogenation and reduction, as confirmed by the in silico analysis. The putative linoleate isomerase and dehydrogenase catalyzing the relevant reactions were identified using the whole genome sequence of $L$. plantarum K25. These two enzymes were shown to bind the LA molecule at their active sites mainly by formation of hydrogen bonding between the acidic group of LA and the proline residues of the enzymes. Glutathione and trypanothione played an important role in the reduction of LA to form the mono-unsaturated and saturated LA analogues, especially the linoleic acid. Further study is required to investigate the role of the conversion from LA to its various analogues by $L$. plantarum physiologically and exploring its potential applications in functional foods and feeds, and their microbiota regulating effects.

\section{Conflicts of Interest}

There is no conflict of interest between the authors.

\section{REFERENCES}

Buccioni A, Decandia M, Minieri S, Molle G, Cabiddu A (2012) Lipid metabolism in the rumen: new insights on lipolysis and biohydrogenation with an emphasis on the role of endogenous plant factors, Anim. Feed Sci. Technol. 174: 1-25. https://doi.org/10.1016/j. anifeedsci.2012.02.009

Alonso L, Cuesta EP, Gilliand SE (2003) Production of free conjugated linoleic acid by Lactobacillus acidophilus and Lactobacillus casei of human intestinal origin. J. Dairy Sci. 86: 1941-1946. https://doi. org/10.3168/jds.S0022-0302(03)73781-3

Barrett E, Ross RP, Fitzgerald GF, Stanton C (2007) Rapid screening method for analyzing the conjugated linoleic acid production capabilities of bacterial cultures. Appl. Environ. Microbiol. 73: 2333-2337. https://doi.org/10.1128/AEM.01855-06

Berlin K, Koren S, Chin CS, Drake JP, Landolin JM, Phillippy AM (2015) Assembling large genomes with single-molecule sequencing and localitysensitive hashing. Nat. Biotechnol. 33: 623-630. https:/9 doi.org/10.1038/nbt.3238

Biasini M, Bienert S, Waterhouse A, Arnold K, Studer G, Schmidt T, Kiefer F, Gallo Cassarino T, Bertoni M, Bordoli L, Schwede T (2014) SWISS-MODEL: modelling protein tertiary and quaternary structure using evolutionary information. Nucleic Acids Research 42: W252-W258. https://doi.org/10.1093/nar/gku340

Zheng CJ, Yoo JS, Lee TG, Cho HY, Kim YH, Kim WG (2005) Fatty acid synthesis is a target for antibacterial activity of unsaturated fatty acids. FEBS Lett. 579: 5157-5162. https://doi.org/10.1016/j. febslet.2005.08.028

Cerning J, Marshall VME (1999) Exopolysaccharides produced by the dairy lactic acid bacteria. Recent Res Dev Microbiol. 3: 195-209

Chung SH, Kim IH, Park HG, Kang HS, Yoon CS, Jeong HY, Choi NJ, Kwon EG, Kim YJ. (2008). Synthesis of conjugated linoleic 
acid by human-derived Bifido bacterium breve LMC 017: utilization as a functional starter culture for milk fermentation. J. Agric. Food Chem. 56: 3311-3316. https://doi.org/10.1021/jf0730789

Greenway DL, Dyke KG (1979) Mechanism of the inhibitory action of linoleic acid on the growth of Staphylococcus aureus. J. Gen. Microbiol. 115: 233-245. https://doi.org/10.1099/00221287-115-1-233

Dassault Systèmes BIOVIA, Discovery Studio Modeling Environment. (2015). https://www.3dsbiovia.com/products/collaborative-science/ biovia-discovery-studio/

De Vuyst L, Degeest B (1999) Heteropolysaccharides from lactic acid bacteria. FEMS Microbiol. Rev. 23: 153-177. https://doi. org/10.1111/j.1574-6976.1999.tb00395.x

Keweloh H, Heipieper HJ (1996) Trans unsaturated fatty acids in bacteria. Lipids 31: 129-137. https://doi.org/10.1016/j. procbio.2019.02.014

Hanwell MD, Curtis DE, Lonie DC, Vandermeerschd T, Zurek E, Hutchison GR (2012) Avogadro: An advanced semantic chemical editor, visualization, and analysis platform. J. Cheminform. 4: 1-17. https:/ / doi.org/10.1186/1758-2946-4-17

Nam IS, Garnsworthy PC (2007) Biohydrogenation of linoleic acid by rumen fungi compared with rumen bacteria. J. Appl. Microbiol. 103: 551-556. https://doi.org 10.1111/j.1365-2672.2007.03317.x

Jiang J, Bjorck L, Fonden R (1998) Production of conjugated linoleic acid by dairy starter cultures. J. Appl. Microbiol. 85: 95-102. https:// doi.org/10.1046/i.1365-2672.1998.00481.x

Kepler CR, Tove SB (1967) Biohydrogenation of unsaturated fatty acids, Purification and properties of a linoleate delta-12-cis, delta11-trans-isomerase from Butyrivibrio fibrisolvens. I. Biol. Chem. 242 5686-5692. http://www.jbc.org/content/242/24/5686

Kepler CR, Tucker WP, Tove SB (1971) Biohydrogenation of unsaturated fatty acids. V. Stereospecificity of proton addition and mechanism of ac tion of linoleic acid 12-cis, $\Delta 11$-trans-isomerase from $B u$ tyrivibrio fibrisolvens. J. Biol. Chem. 246: 2765-2771. https://www.ncbi. nlm.nih.gov/pubmed/7061501

Koren S, Phillippy AM (2015) one chromosome, one contig: complete microbial genomes from long-read sequencing and assembly. Curr. Opin. Microbiol. 23: 110-120. https://doi.org/10.1016/j. mib.2014.11.014

Liavonchanka A, Hornung E, Feussner I, Rudolph MG (2006) Structure and mechanism of the Propionibacterium acnes polyunsaturated fatty acid isomerase. Proc. Natl. Acad. Sci. USA 103: 2576-2581. https://doi.org/10.1073/pnas.0510144103

Liavonchanka A, Rudolph MG, Tittmann K, Hamberg M, Feussner I (2009) On the mechanism of a polyunsaturated fatty acid double bond isomerase from Propionibacterium acnes. J. Biol. Chem. 284: 80058012. https://doi.org/10.1074/jbc.M809060200

Raychowdhury MK, Goswami R, Chakrabarti P (1985) Effect of unsaturated fatty acids in growth inhibition of some penicillin-resistant and sensitive bacteria, J. Appl. Bacteriol. 59: 183-188. https://doi. org/10.1111/j.1365-2672.1985.tb03319.x

Maia MR, Chaudhary LC, Figueres L, Wallace RJ (2007) Metabolism of polyunsaturated fatty acids and their toxicity to the microflora of the rumen. Antonie Van Leeuwenhoek. 91: 303-314. https://doi. org/10.1007/s10482-006-9118-2

Morris GM, Huey R, Lindstrom W, Sanner MF, Belew RK, Goodsell DS (2009) AutoDock4 and AutoDockTools4: Automated docking with selective receptor flexibility. J. Comput. Chem. 30: 2785-2791. https://doi.org/10.1002/jcc.21256

McKain N, Shingfield KJ, Wallace RJ (2010) Metabolism of conjugated linoleic acids and 18: 1 fatty acid by ruminal bacteria: products and mechanisms. Microbiology 156: 579-588. https://doi.org/10.1099/ mic. $0.036442-0$

Nes IF, Yoon S, Diep DB (2007) Ribosomally synthesized antimicrobial peptides (bacteriocins) in lactic acid bacteria: a review. Food Sci. Biotechnol. 16: 675-690. https://doi.org/10.2174/1389450024605409
Ogawa J, Kishino S, Ando A, Sugimoto S, Mihara K, Shimizu S (2005) Production of conjugated fatty acids by lactic acid bacteria. J. Biosci. Bioeng. 100: 355-364. https://doi.org/10.1263/jbb.100.355

Ross Overbeek, Robert Olson, Gordon D Pusch, Gary J. Olsen James J. Davis, Terry Disz, Robert A. Edwards, Svetlana Gerdes, Bruce Parrello, Maulik Shukla, Veronika Vonstein, Alice R. Wattam, Fangfang Xia, Rick Stevens (2014) The SEED and the rapid annotation of microbial genomes using subsystems technology (RAST). Nucleic Acids Res. 42: D206-D214. https://doi.org/10.1093/nar/gkt1226

Peng SS, Deng MD, Grund AD, Rosson R (2007) Purification and characterization of a membrane-bound linoleic acid isomerase from Clostridium sporogenes. Ensyme Microb. Tech. 40: 831-839. https://doi. org/10.1016/j.enzmictec.2006.06.020

Kishino S, Ogawa J, Ando A, Iwashita T, Fujita T, Kawashima H, Shimizu S (2003) Structural analysis of conjugated linoleic acid produced by Lactobacillus plantarum, and factors affecting isomer production. Biosci. Biotechnol. Biochem. 67: 179-182. https://doi. org/10.1271/bbb.67.179

Kishino S, Ogawa J, Yokozeki K, Shimizu S (2011) Linoleic acid isomerase in Lactobacillus plantarum AKU1009a proved to be a multi-component enzyme system requiring oxidoreduction cofactors. Biosci. Biotechnol. Biochem. 75: 318-322. https://doi.org/10.1271/ bbb.100699

Kishino S, Takeuchi M, Park SB, Hirata A, Kitamura N, Kunisawa J, Kiyono H, Iwamoto R, Isobe Y, Arita M, Arai H, Ueda K, Shima J, Takahashi S, Yokozeki K, Shimizu S, Ogawa J (2013) Polyunsaturated fatty acid saturation by gut lactic acid bacteria affecting host lipid composition. Proc. Natl. Acad. Sci. USA 110: 17808-17813. https://doi.org/10.1073/pnas.1312937110

Kishino S, Park SB, Takeuchi M, Yokozeki K, Shimizu S, Ogawa J (2011) Novel multicomponent enzyme machinery in lactic acid bacteria catalyzing $\mathrm{C}=\mathrm{C}$ double bond migration useful for conjugated fatty acid synthesis. Biochem. Biophys. Res. Commun. 416: 188-193. https://doi.org/10.1016/j.bbrc.2011.11.022

Sakurama H, Kishino S, Mihara K, Ando A, Kita K, Takahashi S, Ogawa J (2014) Biohydrogenation of C20 polyunsaturated fatty acids by anaerobic bacteria. J. Lipid Res. 55: 1855-1863. https://doi. org/10.1194/jlr.M045450

Shantha N, Decker EA, Henning B (1993) Comparison of methylation methods for the quantification of Conjugated Linoleic Isomer. J. AOAC Int. 76: 664-649

Tung Y Lin, Chin-Wen Lin, Chien Hsing Lee (1999) Conjugated linoleic acid concentration as affected by lactic cultures and added linoleic acid. Food Chem. 67: 1-5. https://doi.org/10.1631/jzus. B1100072

van Nieuwenhove CP, Oliszewski R, Gonzalez SN, Perez Chaia AB (2007) Conjugated linoleic acid conversion by dairy bacteria cultured in MRS broth and buffalo milk. Lett. Appl. Microbiol. 44: 467-474. https://doi.org/10.1111/j.1472-765X.2007.02135.x

Yunyun Jiang, Jian Zhanga, Xiao Zhaoa, Wen Zhaoa, Zhijian Yub, Chao Chen, Zhennai Yang (2018) Complete genome sequencing of exopolysaccharide-producing Lactobacillus plantarum K25 provides genetic evidence for the probiotic functionality and cold endurance capacity of the strain. Biosci. Biotechnol. Biochem. 82: 1225-1233. https://doi.org/10.1080/09168451.2018.1453293

Yunyun Jiang, Zhennai Yang (2018) Functional and genetic overview of exopolysaccharide produced by Lactobacillus plantarum. J. Funct. Foods. 47: 229-240. https://doi.org/10.1080/09168451.2018.145329 3

Zhang L, Zhang X, Liu CH (2013) Manufacture of Cheddar cheese using probiotic Lactobacillus plantarum K25 and its cholesterol-lowering effects in a mice model. World J. Microbiol. Biotechnol. 29: 127-135. https://doi.org/10.1007/s11274-012-1165-4 